\title{
Nonclassical correlation of polarization-entangled photons in a biexciton-exciton cascade
}

\author{
Sumanta Das and G S Agarwal \\ Department of Physics, Oklahoma State University, Stillwater, OK 74078, USA \\ E-mail: sumanta.das@okstate.edu and agirish@okstate.edu
}

Received 19 August 2008, in final form 7 October 2008

Published 7 November 2008

Online at stacks.iop.org/JPhysB/41/225502

\begin{abstract}
We develop a theoretical model to study the intensity-intensity correlation of polarization-entangled photons emitted in a biexciton-exciton cascade. We calculate the degree of correlation and show how polarization correlations are affected by the presence of dephasing and energy-level splitting of the excitonic states. Our theoretical calculations are in agreement with the recent observation of polarization-dependent intensity-intensity correlations from a single semiconductor quantum dot (Stevenson et al 2006 Nature 439 179). Our model can be extended to study polarization-entangled photon emission in coupled quantum dot systems.
\end{abstract}

(Some figures in this article are in colour only in the electronic version)

\section{Introduction}

Polarization correlations of photons emitted in cascade emission are a well-known phenomenon and numerous theoretical and experimental studies exist in the literature on this subject since the early days of quantum optics [1-6]. Some of the earlier studies were motivated in testing generalized Bell's inequalities [2], the existence of hidden variables and whether quantum mechanics was a non-local theory or not $[3,4]$, following the question raised by Einstein, Podolsky and Rosen [7]. In recent times, polarization-correlated photon pairs have become important in the field of quantum information science due to their entangled nature. Moreover many applications of quantum information, such as quantum key distribution [8], efficient optical quantum computing [9], long-distance quantum communication using quantum repeaters [10] and implementation of quantum telecommunication schemes [11] require single-photon pairs per cycle. This requirement of entangled photon pairs per cycle of excitation could be satisfied by cascade emission from a single-atom or atom-like systems such as semiconductor quantum dots, provided one gets over the inherent asymmetries. Recently, such cascade emission has been reported for semiconductor quantum dots [12-15]. It was further seen that polarization entanglement of the emitted photon pairs was degraded by the presence of energy-level splitting of the intermediate excitonic states and any incoherent process that leads to a population transfer between the two intermediate excitonic states [14-16]. Moreover, dephasing arising due to interaction of the quantum dot with its solid-state environment can also degrade the entanglement [17]. Some recent studies have also shown how the fidelity of entanglement depends on excitonic-level splitting [18] and the dynamics of the incoherent dephasing [19]. Different methods have been proposed to reduce and control the incoherent dephasing and energy-level splitting of the excitonic states thereby preserving the entanglement in the system [14-16, 18, 20-22]. Further methods to enhance the generated entanglement by coupling the quantum dot to a micro-cavity have also been proposed $[23,24]$. As quantum dot systems are of great importance for future applications in quantum information science, a clear yet simple model for understanding the effects of all these different decoherence mechanisms on the dynamics of the system is required. Thus we develop, in this paper, a simple theoretical model to analytically study the influence of different decoherence mechanisms and the intermediate state splitting on the generation of polarization-entangled photon pairs in cascade emission. 
$|\alpha\rangle$

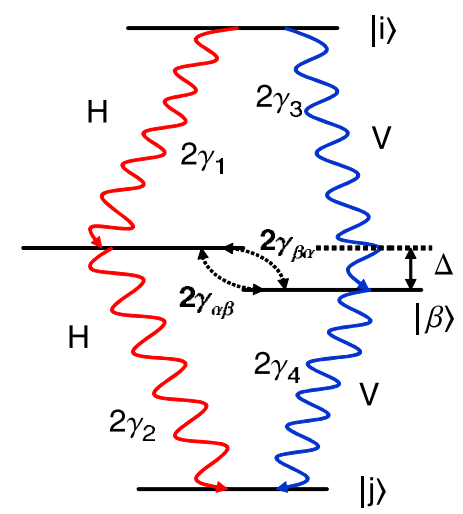

Figure 1. Schematic diagram of a four-level cascade system. Here $\mathrm{H}$ and $\mathrm{V}$ refer to horizontally and vertically polarized photon emission. $\Delta$ is the energy-level separation of the intermediate states and $\gamma$ 's are the spontaneous emission rates given by $\gamma_{k}=2 \omega_{k l}^{3}\left|\vec{d}_{k l}\right|^{2} / 3 \hbar c^{3}$. The incoherent dephasing rates of the intermediate states are given by $2 \gamma_{\alpha \beta}$ and $2 \gamma_{\beta \alpha}$ respectively.

\section{Model}

We consider a four-level system undergoing cascade emission as our model. We show a schematic diagram of such a cascade in figure 1. The excited state $|i\rangle$ and the intermediate states $|\alpha\rangle,|\beta\rangle$ would correspond to the biexcitonic and optically active excitonic states respectively in a quantum dot. Further $|j\rangle$ is taken to be the ground state. Here $2 \gamma=2\left(\gamma_{1}+\gamma_{3}\right)$ is the total spontaneous emission rate of the state $|i\rangle, 2 \gamma_{2}$ and $2 \gamma_{4}$ are the spontaneous emission rates of the states $|\alpha\rangle$ and $|\beta\rangle$ respectively and $2 \gamma_{\beta \alpha}\left(2 \gamma_{\alpha \beta}\right)$ is the incoherent dephasing rate of the state $|\alpha\rangle(|\beta\rangle)$. The energy-level splitting of the intermediate state is given by $\Delta$. In this type of four-level cascade scheme there are two decay paths for the excited state, $|i\rangle \rightarrow|\alpha\rangle \rightarrow|j\rangle$ and $|i\rangle \rightarrow|\beta\rangle \rightarrow|j\rangle$. The generation of entanglement in these schemes is attributed to the fact that these decay paths can become indistinguishable. The eigenbasis of this system is formed by the four states $(\{|i\rangle\},\{|\alpha\rangle\},\{|\beta\rangle\},\{|j\rangle\})$. In this basis, the radiative transition from the excited state generates collinearly polarized photons with linear polarizations along two orthogonal directions denoted by $\mathrm{H}$ (horizontal) and $\mathrm{V}$ (vertical). When the states $|\alpha\rangle$ and $|\beta\rangle$ are degenerate, the decay paths become indistinguishable and we get a maximally entangled twophoton state $[7,12]$

$$
|E\rangle=\frac{1}{\sqrt{2}}\left(\left|H_{1} H_{2}\right\rangle+\left|V_{1} V_{2}\right\rangle\right) .
$$

In practical systems such as atoms and quantum dots these levels are usually non-degenerate and hence the entanglement of the emitted photon pairs depends completely on the degree of degeneracy and dynamics of these intermediate states. In our model, we have taken them to be non-degenerate and study the effect of such intermediate-level splitting on the correlation of the emitted photon pairs. To understand the effect of incoherent dephasing and energy-level splitting of the excitonic state on the dynamics of emitted photon pairs from the cascade, we need to study the two-time second-order correlations. This is given by

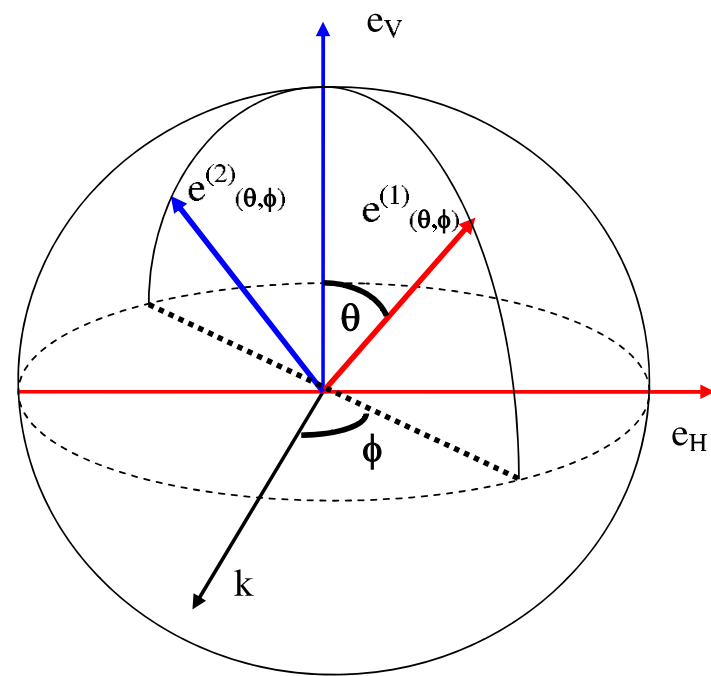

Figure 2. Schematic diagram for the orientation of the polarization unit vectors. Here, $\mathbf{e}_{H}$ and $\mathbf{e}_{V}$ correspond to horizontal and vertical polarization respectively. $\mathbf{e}^{(1)}$ and $\mathbf{e}^{(2)}$ are two arbitrary orthogonal pair of polarization unit vectors.

$$
\begin{gathered}
\langle I I\rangle=\left\langle\hat{\epsilon}_{\left(\theta_{1}, \phi_{1}\right)}^{*} \cdot \vec{E}^{-}(\vec{r}, t) \hat{\epsilon}_{\left(\theta_{2}, \phi_{2}\right)}^{*} \cdot \vec{E}^{-}(\vec{r}, t+\tau)\right. \\
\left.: \hat{\epsilon}_{\left(\theta_{2}, \phi_{2}\right)} \cdot \vec{E}^{+}(\vec{r}, t+\tau) \hat{\epsilon}_{\left(\theta_{1}, \phi_{1}\right)} \cdot \vec{E}^{+}(\vec{r}, t)\right\rangle,
\end{gathered}
$$

where $\langle I I\rangle$ stands for the two-time polarization-angledependent intensity-intensity correlation $\left\langle I_{\left(\theta_{2}, \phi_{2}\right)}(\vec{r}, t+\right.$ $\left.\tau) I_{\left(\theta_{1}, \phi_{1}\right)}(\vec{r}, t)\right\rangle$. Further, $E^{+}(\vec{r}, t)\left(E^{-}(\vec{r}, t)\right)$ is the positive (negative) frequency part of the quantized electric field operator at a point $\vec{r}$ in the far-field zone and $\hat{\epsilon}_{(\theta, \phi)}$ is the polarization unit vector of the measured radiation at the detector along any arbitrary direction given by $(\theta, \phi) . \hat{\epsilon}_{(\theta, \phi)}$ 's are related to the linear polarization unit vectors $\hat{\epsilon}_{H}, \hat{\epsilon}_{V}$ (where $\mathrm{H}$ stands for horizontal and $\mathrm{V}$ for vertical) by

$$
\left[\begin{array}{c}
\hat{\epsilon}_{(\theta, \phi)}^{(1)} \\
\hat{\epsilon}_{(\theta, \phi)}^{(2)}
\end{array}\right]=\left[\begin{array}{cc}
\cos \theta & \mathrm{e}^{-\mathrm{i} \phi} \sin \theta \\
-\mathrm{e}^{\mathrm{i} \phi} \sin \theta & \cos \theta
\end{array}\right]\left[\begin{array}{c}
\hat{\epsilon}_{H} \\
\hat{\epsilon}_{V}
\end{array}\right]
$$

and these satisfy the relation $\left(\hat{\epsilon}_{(\theta, \phi)}^{(1)} \cdot \hat{\epsilon}_{(\theta, \phi)}^{(2) *}\right)=0$. The above relation can be understood as an unitary transformation between a basis defined by the linear polarization unit vectors and a basis defined by $\hat{\epsilon}^{(1)}$ and $\hat{\epsilon}^{(2)}$ (see figure 2). In the experimental setup, the angles $\theta, \phi$ would correspond to the orientation of the optic axis of a half/quarter wave plate to the direction of propagation of the emitted radiation. Let us now consider for simplicity that both the levels $|\alpha\rangle$ and $|\beta\rangle$ in figure 1 have the same incoherent dephasing rates, i.e. $\gamma_{\alpha \beta}=$ $\gamma_{\beta \alpha}$. Further, we assume that the spontaneous decay rates of the intermediate levels are also equal. Such assumptions are well justified as they do not influence the dynamics of the system significantly and yet leads to a simplified form of the second-order correlation, thereby providing a better understanding of the problem. Under the above assumptions and for $\phi_{1}=\phi_{2}=0$ the form of the two-time polarizationangle-dependent intensity-intensity correlation is found to be

$$
\begin{aligned}
& \left\langle I\left(\theta_{2}, t+\tau\right) I\left(\theta_{1}, t\right)\right\rangle=\left(\frac{\omega_{0}}{c}\right)^{8} \frac{1}{2 r^{4}} \mathcal{D}_{1}^{2} \mathcal{D}_{2}^{2}\langle\mid i\rangle\left\langle\left. i\right|_{t}\right\rangle \\
& \quad \times\left\{\mathrm{e}^{-2 \gamma_{2} \tau}+\cos 2 \theta_{1} \cos 2 \theta_{2} \mathrm{e}^{-2\left(\gamma_{2}+2 \gamma_{\alpha \beta}\right) \tau}\right. \\
& \left.\quad+\sin 2 \theta_{1} \sin 2 \theta_{2} \mathrm{e}^{-2\left(\gamma_{2}+\gamma_{\alpha \beta}\right) \tau} \cos (\Delta \tau)\right\},
\end{aligned}
$$




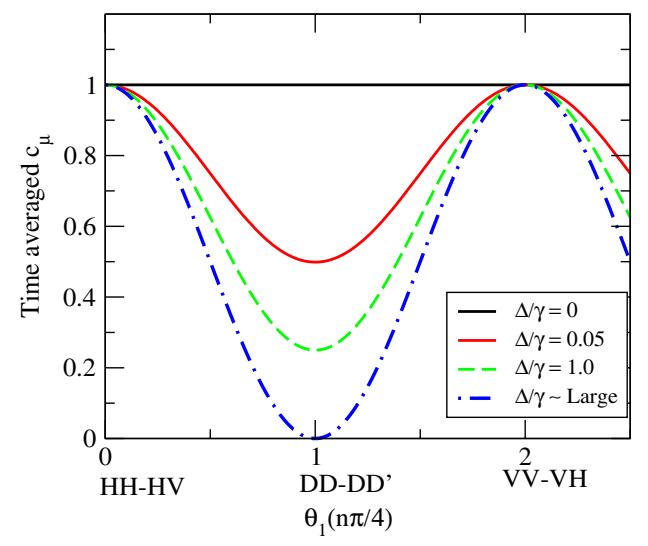

(a)

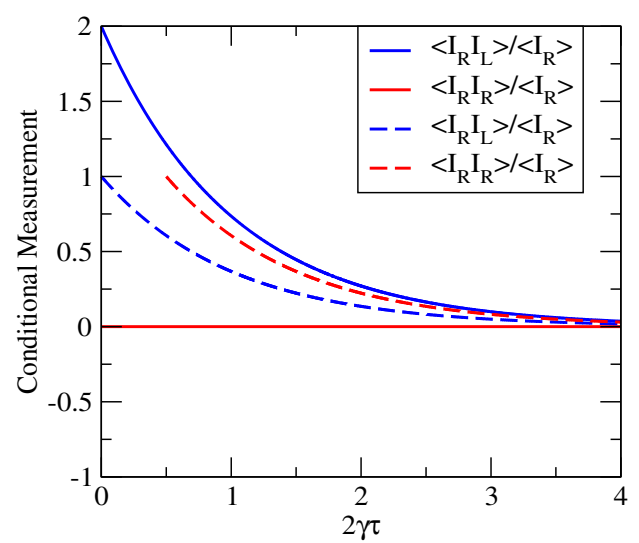

(b)

Figure 3. (a) Degree of correlation averaged over time as a function of basis angle, for different excitonic-level splitting $\Delta$. H, D, $D^{\prime}$, V stands for horizontal, diagonal, orthodiagonal and vertical polarization basis respectively. Here we have considered zero dephasing of the excitonic states. (b) Conditional measurement of intensity-intensity correlation in the circular basis. The red curve corresponds to co-polarized $\left(\theta_{1}=\theta_{2}=\pi / 4, \phi_{1}=\phi_{2}=-\pi / 2\right)$ photons and the blue for cross-polarized $\left(\theta_{1}=\theta_{2}=\pi / 4, \phi_{1}=-\pi / 2, \phi_{2}=\pi / 2\right)$ ones. The solid curve is for $\Delta=0$ and the broken one for $\Delta \sim$ large. Here $\mathrm{R}$ and $\mathrm{L}$ stands for right and left circular polarization. The $\mathrm{R}-\mathrm{R}$ correlation curve in the case of large splitting is time shifted for better comparison to the R-L correlation. All parameters are normalized with respect to $\gamma$.

where $\mathcal{D}_{1}=\left|\vec{d}_{\alpha i}\right|=\left|\vec{d}_{\beta i}\right|$ and $\mathcal{D}_{2}=\left|\vec{d}_{j \alpha}\right|=\left|\vec{d}_{j \beta}\right|$. The above simple form of the second-order correlation has been derived to match our theoretical analysis to that of the experimental results [15]. For details of the mathematical analysis leading to the generalized form of the two-time intensity-intensity correlation the reader is referred to section 4 of this paper. One can clearly see from equation (4) that the second-order correlation is profoundly influenced by both the incoherent dephasing rates as well as the energy-level splitting of the intermediate states. Note further that in the presence of small $\Delta$ this becomes equivalent to the second-order correlations measured in $[3,4]$. Next, we define a quantity the degree of correlation $c_{\mu}$ as

$$
c_{\mu}=\frac{\left\langle I_{\mu} I_{\mu}\right\rangle-\left\langle I_{\mu} I_{\mu^{\prime}}\right\rangle}{\left\langle I_{\mu} I_{\mu}\right\rangle+\left\langle I_{\mu} I_{\mu^{\prime}}\right\rangle}
$$

where $\mu, \mu^{\prime}$ stands for mutually orthogonal polarization basis. The degree of correlation varies between +1 and -1 , where +1 represents perfect correlation ( -1 for anti-correlation) and 0 represents no polarization correlation.

\section{Results and discussion}

\subsection{Effect of excitonic-level splitting on the correlation}

In figure 3(a), we show how the time-averaged degree of correlation varies with the basis angle for different values of splitting $\Delta$ of the excitonic levels. Note that here the excitoniclevel dephasing $\gamma_{\alpha \beta}$ has been taken to be zero. We see that the degree of correlation is independent of the polarization basis when $\Delta=0$ and takes a value $c_{\mu}=1$. This corresponds to a perfect polarization correlation among the emitted photons. From the expression of $c_{\mu}$ it is clear that this can happen only when the cross-polarized correlations vanish and the emitted photons are perfectly co-polarized. One can even see this explicitly from equation (4) by putting the values of $\theta_{1}, \theta_{2}=\theta_{1}+\pi / 2$ for $\mathrm{H}-\mathrm{V}, \mathrm{D}-\mathrm{D}^{\prime}$ and $\mathrm{V}-\mathrm{H}$ basis, where $\mathrm{H}, \mathrm{V}, \mathrm{D}$ and $\mathrm{D}^{\prime}$ stands for horizontal, vertical, diagonal and orthodiagonal polarization basis, respectively. Further, as the cross-polarized correlations are absent the pair of photons emitted in one excitation cycle can take either of the two paths $|i\rangle \rightarrow|\alpha\rangle \rightarrow|j\rangle$ or $|i\rangle \rightarrow|\beta\rangle \rightarrow|j\rangle$ thus making these paths indistinguishable. As a consequence, we do not get the 'Welcher Weg' or which path information thereby making the final state of the emitted photon pair entangled in both the linear and diagonal polarization basis. The generated entangled states can hence be written as $1 / \sqrt{2}(|H H\rangle+|V V\rangle)$ and $1 / \sqrt{2}\left(|D D\rangle+\left|D^{\prime} D^{\prime}\right\rangle\right)$ for the rectilinear and diagonal basis respectively. Note further that in this case perfect anticorrelation $\left(c_{\mu}=-1\right)$ is expected for measurement in the circularly polarized basis with the entangled state given by $1 / \sqrt{2}(|R L\rangle+|L R\rangle)$. Thus one should get perfectly crosspolarized photons as the co-polarized correlations vanish in this basis. This is exactly what we get from the general expression of equation (4) (see section 4, equation (13)) and is shown by the solid curves in figure 3(b). Further in figure 3(a) we see that the degree of correlation is practically independent of the excitonic-level splitting $\Delta$ in the rectilinear basis. As we change our polarization basis the effect of $\Delta$ becomes significant. In the diagonal basis, for example, with the increase in level splitting the degree of correlation gradually decreases and eventually vanishes. In the presence of $\Delta$, the cross-polarization does not vanish and we have path information for the emitted photons when we measure the second-order correlations, thus destroying any entanglement in the system. The behaviour of the correlations in the circular basis in the presence of large excitonic-level splitting is shown by the broken curves in figure 3(b). One can clearly see that there is no polarization correlation at all for large $\Delta$. The sinusoidal behaviour of $c_{\mu}$ for nonzero value of $\Delta$ as seen in figure 3(a) is in agreement with the classical linear 


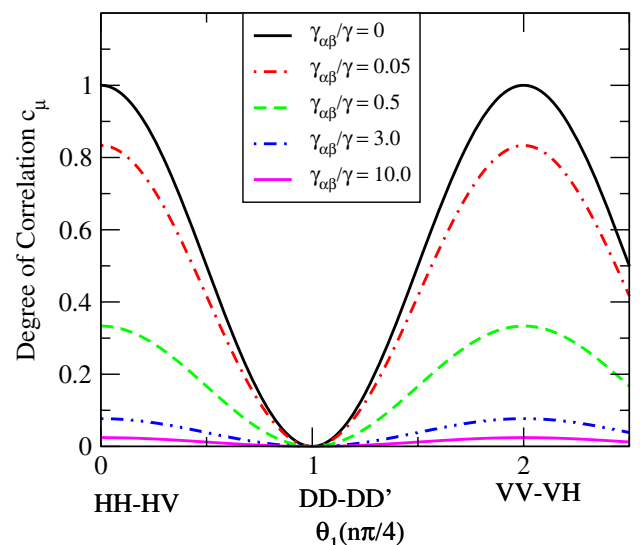

(a)

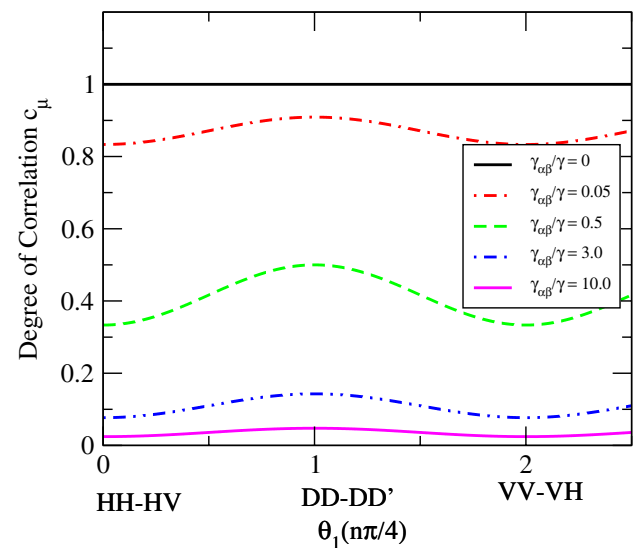

(b)

Figure 4. (a) Degree of correlation averaged over time as a function of basis angle, for large $\Delta$ and different incoherent dephasing rates $\gamma_{\alpha \beta}$ of the intermediate level. Here we have assumed that both the intermediate states dephase at same rate, i.e. $\gamma_{\alpha \beta}=\gamma_{\beta \alpha}$. H, D, D', V stands for horizontal, diagonal, orthodiagonal and vertical basis respectively. (b) Same as (a) for $\Delta=0$.

polarization correlation behaviour. Note that our theoretical results are in agreement to experimentally observed data [15]. It should be noted that in our analysis we have concentrated on the calculation of the quantum correlation $c_{\mu}$. This was also measured in the experiment of Stevenson et al. We have not examined measures of entanglement like concurrence. This is because if $\Delta$ the intermediate state exciton splitting is nonzero then horizontal and vertical photons have different frequencies which amounts to saying that we have for nonzero $\Delta$ quantum states which are characterized by two different parameters and measures of entanglement in such situations do not exist.

\subsection{Effect of decoherence on the correlation}

In figures 4(a) and (b), we show how the incoherent dephasing of the intermediate excitonic states affect the time-averaged degree of correlations $c_{\mu}$ when excitonic states are nondegenerate $(\Delta \neq 0)$ and degenerate $(\Delta=0)$ respectively. Note that here we have assumed that both the intermediate states have same dephasing rates. One can clearly see that the effect is different for different measurement basis. The degree of polarization correlation for example in the rectilinear basis decreases with increasing dephasing irrespective of whether the excitonic states are non-degenerate or degenerate. For large dephasing rates the emitted photon pairs become almost un-correlated in their polarization. This is attributed to the presence of significant cross-polarized correlation for large dephasing rates of the intermediate states. The incoherent dephasing of the intermediate levels causes a incoherent population transfer among the states $|\alpha\rangle$ and $|\beta\rangle$ thereby allowing the second photon to be emitted with orthogonal polarization to the first one. In the diagonal basis, on the other hand, the dephasing does not affect the correlation at all for large $\Delta$ but significantly decreases the correlation when $\Delta=0$ for large dephasing rates. So we see that in diagonal basis even when the intermediate levels are degenerate we can still have significant cross-correlation if there is some incoherent relaxation process by which they can get coupled. This in turn spoils the quantum correlation in the system

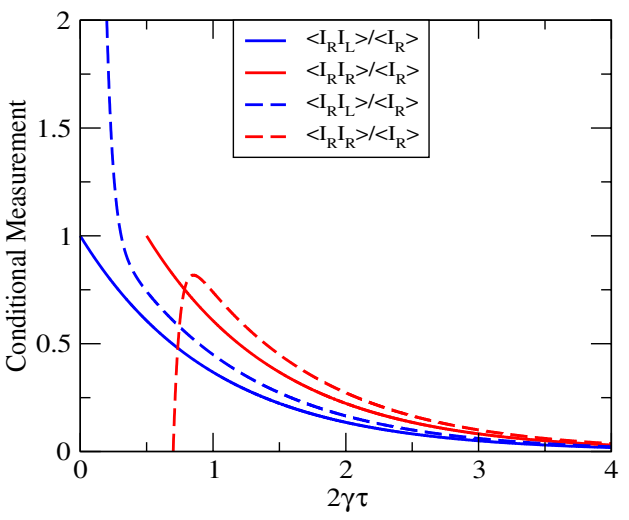

Figure 5. Conditional measurement of intensity-intensity correlation in the circular basis for incoherent dephasing $\gamma_{\alpha \beta} / \gamma=10$. The red curve corresponds to co-polarized $\left(\theta_{1}=\theta_{2}=\pi / 4, \phi_{1}=\phi_{2}=-\pi / 2\right)$ photons and the blue for cross-polarized $\left(\theta_{1}=\theta_{2}=\pi / 4, \phi_{1}=-\pi / 2, \phi_{2}=\pi / 2\right)$ ones. The solid curve is for $\Delta \sim$ large and broken one for $\Delta=0$. Here $\mathrm{R}$ and $\mathrm{L}$ stand for right and left circular polarization. The $\mathrm{R}-\mathrm{R}$ correlation curves are time shifted for better comparison to the $\mathrm{R}-\mathrm{L}$ correlation.

as can be seen clearly from figure 4(b). In figure 5, we show how the correlations behave in the circular basis in the presence of a large dephasing rate $\left(\gamma_{\alpha \beta} / \gamma=10\right)$ for both non-degenerate and degenerate intermediate states. We find that in the circular basis decoherence arising due to the incoherent dephasing does not affect the degree of correlation of the emitted photons when $\Delta$ is large. Further we find that for degenerate intermediate levels, even though the degree of correlation $c_{\mu}=-1$ for zero time delay, it vanishes at all later time in the presence of the large dephasing. Thus the decoherence makes the perfectly anti-correlated photons completely uncorrelated. The incoherent relaxation process discussed by us here is practically present in the biexcitonicexcitonic cascade in quantum dots $[17,19]$. Thus we have shown by a simple model how the decoherence arising due to such incoherent processes would strongly affect the quantum correlations. 


\section{Detail derivation of the intensity-intensity correlation}

Our model consists of a biexcitonic state and two excitonic states labelled as $|i\rangle$ and $|\alpha\rangle,|\beta\rangle$ respectively. The equilibrium state is labelled as $|j\rangle$. The biexcitonic state decays by emission of either a horizontally $(\mathrm{H})$ polarized photon $(|i\rangle \rightarrow$ $|\alpha\rangle)$ or a vertically $(\mathrm{V})$ polarized photon $(|i\rangle \rightarrow|\beta\rangle)[12,13]$. The excitonic state $|\alpha\rangle(|\beta\rangle)$ decays to the equilibrium state $|j\rangle$ by emission of a $\mathrm{H}(\mathrm{V})$ - polarized photon. The two excitonic states have an energy difference of $\hbar \Delta$. Note that the splitting of the excitonic state in quantum dots arises due to anisotropic electron-hole exchange interactions [25, 26]. Figure 1 shows a schematic diagram of our model. The eigenbasis of this system is formed by the four states $(\{|i\rangle\},\{|\alpha\rangle\},\{|\beta\rangle\},\{|j\rangle\})$. In this basis, the unperturbed Hamiltonian $\mathcal{H}$ is given by

$$
\mathcal{H}=\sum_{k} \hbar \omega_{k}|k\rangle\langle k|,
$$

where $\hbar \omega_{k}$ is the energy of the four levels $(k=i, \alpha, \beta, j)$. Note that this kind of energy-level scheme has been extensively used to study the cascade emission in quantum dots [14, 16-19]. The spontaneous emission and dephasing effects in the system are incorporated via a master equation technique [27] under the Born, Markov and rotating wave approximations and is given by

$$
\begin{aligned}
\mathcal{L} \rho & =-\gamma\left\{S_{i i}, \rho\right\}-\gamma_{2}\left\{S_{\alpha \alpha}, \rho\right\}-\gamma_{4}\left\{S_{\beta \beta}, \rho\right\}-\gamma_{\beta \alpha}\left\{S_{\alpha \alpha}, \rho\right\} \\
& -\gamma_{\alpha \beta}\left\{S_{\beta \beta}, \rho\right\}+2\left(\gamma_{1} \rho_{i i} S_{\alpha \alpha}+\gamma_{3} \rho_{i i} S_{\beta \beta}+\gamma_{2} \rho_{\alpha \alpha} S_{j j}\right. \\
& \left.+\gamma_{4} \rho_{\beta \beta} S_{j j}\right)+2\left(\gamma_{\beta \alpha} \rho_{\alpha \alpha} S_{\beta \beta}+\gamma_{\alpha \beta} \rho_{\beta \beta} S_{\alpha \alpha}\right) .
\end{aligned}
$$

Here $2 \gamma=2\left(\gamma_{1}+\gamma_{3}\right)$ is the total spontaneous emission rate of the biexcitonic state $|i\rangle, 2 \gamma_{2}\left(2 \gamma_{4}\right)$ and $2 \gamma_{\beta \alpha}\left(2 \gamma_{\alpha \beta}\right)$ are the spontaneous emission rate and incoherent dephasing rate of the excitonic state $|\alpha\rangle(|\beta\rangle)$ (see figure 1). Such incoherent dephasing arises in quantum dots due to its interaction with the solid-state environment (in the form of spin-flip processes or phonon scattering) [17]. The curly bracket $\{. ., .$.$\} stands for the$ anti-commutator and $S_{k l}=|k\rangle\langle l|\left(S_{k l}^{\dagger}=|l\rangle\langle k|\right)$ is the atomic lowering (raising) operator which follow the simple angular momentum commutation relations. To study the dynamical evolution of this four-level cascade system we solve for the time evolution of the density operator which is given by

$$
\frac{\partial \rho}{\partial t}=-\frac{\mathrm{i}}{\hbar}[\mathcal{H}, \rho]+\mathcal{L} \rho .
$$

We will set the energy of the state $|j\rangle$ equal to zero henceforth. On substituting equations (6) and (7) into (8) and solving for the population terms we get

$$
\begin{aligned}
& p_{i}(t)=\mathrm{e}^{-2 \gamma t} p_{i}(0) ; \quad p_{i}=\rho_{i i}-R / 2 \gamma \\
& \rho_{\alpha \beta}(t)=\mathrm{e}^{-\left(a_{0}-i \Delta\right) t} \rho_{\alpha \beta}(0) ; \\
& \rho_{\alpha \alpha}(t)=\mathrm{e}^{-a_{0} t}\left(\cosh (A t)+\frac{\Gamma_{a}}{A} \sinh (A t)\right) \rho_{\alpha \alpha}(0) \\
& +2 \mathrm{e}^{-a_{0} t} \frac{\gamma_{\alpha \beta}}{A} \sinh (A t) \rho_{\beta \beta}(0) \\
& +\frac{R}{2 \gamma} C(t)+p_{i}(0) \mathrm{e}^{-2 \gamma t} D(t)
\end{aligned}
$$

$$
\begin{aligned}
\rho_{\beta \beta}(t) & =\mathrm{e}^{-a_{0} t}\left(\cosh (A t)-\frac{\Gamma_{a}}{A} \sinh (A t)\right) \rho_{\beta \beta}(0) \\
+ & 2 \mathrm{e}^{-a_{0} t} \frac{\gamma_{\beta \alpha}}{A} \sinh (A t) \rho_{\alpha \alpha}(0) \\
+ & \frac{R}{2 \gamma} F(t)+p_{i}(0) \mathrm{e}^{-2 \gamma t} K(t) .
\end{aligned}
$$

Here $R$ signifies a constant feeding of population into the state $|i\rangle$ from some arbitrary state $|n\rangle$. Note that in our model we are only concerned with the dynamics of the cascade decay once the upper level is populated, and thereby do not consider explicitly the pumping of the biexciton state $|i\rangle$. Further the excitonic-level splitting $\Delta=\omega_{\alpha}-\omega_{\beta}$, $a_{0}=\left(\gamma_{2}+\gamma_{4}+\gamma_{\alpha \beta}+\gamma_{\beta \alpha}\right), \Gamma_{a}=\left(\gamma_{4}-\gamma_{2}+\gamma_{\alpha \beta}-\gamma_{\beta \alpha}\right)$ and $A=\sqrt{\Gamma_{a}^{2}+4 \gamma_{\alpha \beta} \gamma_{\beta \alpha}}$. The time-dependent coefficients $C, D, F$ and $K$ are given by

$$
\begin{aligned}
C(t) & =\left(2 \gamma_{1}\left(1+\frac{\Gamma_{a}}{A}\right)+4 \frac{\gamma_{3} \gamma_{\alpha \beta}}{A}\right) \frac{1-\mathrm{e}^{-\left(a_{0}-A\right) t}}{2\left(a_{0}-A\right)} \\
+ & (A \rightarrow-A), \\
D(t) & =\left(2 \gamma_{1}\left(1+\frac{\Gamma_{a}}{A}\right)+4 \frac{\gamma_{3} \gamma_{\alpha \beta}}{A}\right) \frac{1-\mathrm{e}^{-\left(a_{0}-A-2 \gamma\right) t}}{2\left(a_{0}-A-2 \gamma\right)} \\
& +(A \rightarrow-A), \\
F(t) & =\left(2 \gamma_{3}\left(1-\frac{\Gamma_{a}}{A}\right)+4 \frac{\gamma_{1} \gamma_{\beta \alpha}}{A}\right) \frac{1-\mathrm{e}^{-\left(a_{0}-A\right) t}}{2\left(a_{0}-A\right)} \\
+ & (A \rightarrow-A), \\
K(t) & =\left(2 \gamma_{3}\left(1-\frac{\Gamma_{a}}{A}\right)+4 \frac{\gamma_{1} \gamma_{\beta \alpha}}{A}\right) \frac{1-\mathrm{e}^{-\left(a_{0}-A-2 \gamma\right) t}}{2\left(a_{0}-A-2 \gamma\right)} \\
+ & (A \rightarrow-A) .
\end{aligned}
$$

The effect of non-degenaracy of the excitonic states and their incoherent dephasing on the dynamical evolution of the system shows up if one studies the two-time nonclassical second-order correlation defined in equation (2). For our four-level system the explicit form of the positive frequency part of the electricfield operator is given by [27]

$$
\begin{aligned}
& \vec{E}^{(+)}(\vec{r}, t)=\vec{E}_{0}^{(+)}(\vec{r}, t)-\left(\frac{\omega_{0}}{c}\right) \frac{1}{r}\left([ \hat { n } \times ( \hat { n } \times \vec { d } _ { \alpha i } ) ] | \alpha \rangle \left\langle\left.i\right|_{t}\right.\right. \\
& +\left[\hat{n} \times\left(\hat{n} \times \vec{d}_{\beta i}\right)\right]|\beta\rangle\left\langle\left. i\right|_{t}+\left[\hat{n} \times\left(\hat{n} \times \vec{d}_{j \alpha}\right)\right] \mid j\right\rangle\left\langle\left.\alpha\right|_{t}\right. \\
& +\left[\hat{n} \times\left(\hat{n} \times \vec{d}_{j \beta}\right)\right]|j\rangle\left\langle\left.\beta\right|_{t}\right) .
\end{aligned}
$$

Finally, using equation (11) in (2) we get the general form of the two-time intensity-intensity correlation

$$
\begin{aligned}
\langle I I\rangle & =\left(\frac{\omega_{0}}{c}\right)^{8} \frac{1}{r^{4}}\left\{\left\langle\left[( \hat { \epsilon } _ { H } \cdot \vec { d } _ { \alpha i } ) ^ { * } \operatorname { c o s } \theta _ { 1 } | i \rangle \left\langle\left.\alpha\right|_{t}\right.\right.\right.\right. \\
& +\left(\hat{\epsilon}_{V} \cdot \vec{d}_{\beta i}\right)^{*} \mathrm{e}^{\mathrm{i} \phi_{1}} \sin \theta_{1}|i\rangle\left\langle\left.\beta\right|_{t}\right] \\
& \times\left(| \hat { \epsilon } _ { H } \cdot \vec { d } _ { j \alpha } | ^ { 2 } \operatorname { c o s } ^ { 2 } \theta _ { 2 } | \alpha \rangle \langle \alpha | _ { t + \tau } + | \hat { \epsilon } _ { V } \cdot \vec { d } _ { j \beta } | ^ { 2 } \operatorname { s i n } ^ { 2 } \theta _ { 2 } | \beta \rangle \left\langle\left.\beta\right|_{t+\tau}\right.\right. \\
& +\mathrm{e}^{-\mathrm{i} \phi_{2}}\left(\hat{\epsilon}_{H} \cdot \vec{d}_{j \alpha}\right)^{*}\left(\hat{\epsilon}_{V} \cdot \vec{d}_{j \beta}\right) \cos \theta_{2} \sin \theta_{2}|\alpha\rangle\left\langle\left.\beta\right|_{t+\tau}\right. \\
& +\mathrm{e}^{\mathrm{i} \phi_{2}}\left(\hat{\epsilon}_{H} \cdot \vec{d}_{j \alpha}\right)\left(\hat{\epsilon}_{V} \cdot \vec{d}_{j \beta}\right)^{*} \cos \theta_{2} \sin \theta_{2}|\beta\rangle\left\langle\left.\alpha\right|_{t+\tau}\right) \\
& \times\left[( \hat { \epsilon } _ { H } \cdot \vec { d } _ { \alpha i } ) \operatorname { c o s } \theta _ { 1 } | \alpha \rangle \left\langle\left.i\right|_{t}\right.\right. \\
& \left.\left.+\left(\hat{\epsilon}_{V} \cdot \vec{d}_{\beta i}\right) \mathrm{e}^{-\mathrm{i} \phi_{1}} \sin \theta_{1}|\beta\rangle\left\langle\left. i\right|_{t}\right]\right\rangle\right\} .
\end{aligned}
$$

The two-time correlation function that appears in equation (12) is evaluated by invoking the quantum regression theorem [28] and equation (9). Finally, we get 


$$
\begin{aligned}
\langle I I\rangle & =\left(\frac{\omega_{0}}{c}\right)^{8} \frac{1}{4 r^{4}} \mathcal{D}_{1}^{2} \mathcal{D}_{2}^{2}\langle\mid i\rangle\left\langle\left. i\right|_{t}\right\rangle\left\{f_{1}(\tau)+w_{1}(\tau)+f_{2}(\tau)\right. \\
& +w_{2}(\tau)+\left(\cos 2 \theta_{1}+\cos 2 \theta_{2}\right)\left(f_{1}(\tau)-w_{2}(\tau)\right) \\
& +\left(\cos 2 \theta_{1}-\cos 2 \theta_{2}\right)\left(w_{1}(\tau)-f_{2}(\tau)\right) \\
& +\cos 2 \theta_{1} \cos 2 \theta_{2}\left(f_{1}(\tau)+w_{2}(\tau)-f_{2}(\tau)-w_{1}(\tau)\right) \\
& \left.+\sin 2 \theta_{1} \sin 2 \theta_{2}\left(\mathrm{e}^{-\mathrm{i}\left(\phi_{1}+\phi_{2}\right)} u(\tau)+\mathrm{e}^{\mathrm{i}\left(\phi_{1}+\phi_{2}\right)} u^{*}(\tau)\right)\right\} .
\end{aligned}
$$

Here $\mathcal{D}_{1}=\left|\vec{d}_{\alpha j}\right|=\left|\vec{d}_{\beta j}\right|$ and $\mathcal{D}_{2}=\left|\vec{d}_{j \alpha}\right|=\left|\vec{d}_{j \beta}\right|$. The $f$ 's, $w$ 's and $u$ are found from the solutions of the density matrix equations (9) and are given by

$$
\begin{aligned}
& f_{1}(\tau)=\mathrm{e}^{-a_{0} \tau}\left(\cosh (A \tau)+\frac{\Gamma_{a}}{A} \sinh (A \tau)\right), \\
& f_{2}(\tau)=2 \mathrm{e}^{-a_{0} \tau} \frac{\gamma_{\alpha \beta}}{A} \sinh (A \tau), \\
& w_{1}(\tau)=2 \mathrm{e}^{-a_{0} \tau} \frac{\gamma_{\beta \alpha}}{A} \sinh (A \tau), \\
& w_{2}(\tau)=\mathrm{e}^{-a_{0} \tau}\left(\cosh (A \tau)-\frac{\Gamma_{a}}{A} \sinh (A \tau)\right), \\
& u(\tau)=\mathrm{e}^{-\left(a_{0}-i \Delta\right) \tau} .
\end{aligned}
$$

Equation (13) gives the most general form of the twotime intensity-intensity correlations for arbitrary polarization directions and for any system undergoing a cascade emission. In the special case of $\phi_{1}=\phi_{2}=0, \gamma_{\alpha \beta}=\gamma_{\beta \alpha}$ and $\gamma_{2}=\gamma_{4}$ this reduces to the simplified result (4) of section (2).

\section{Conclusions}

In conclusion, we have developed a simple theory to understand how the dephasing and energy-level splitting of the excitonic states can affect polarization entanglement of photons emitted in a biexciton-exciton cascade. We have also shown how these effects are important in determining whether the emitted photon pairs are classically correlated or entangled in different polarization basis. Further, we have shown that our theoretical calculation is in agreement with the experimental results found in context to such cascade emissions in quantum dots. As a future prospect it would be interesting to extend the method of the present paper to a system of coupled dots or a dot in the micro-cavity.

\section{Acknowledgments}

This work was supported by NSF grant nos PHYS-0653494 and CCF-0829860.

\section{References}

[1] Kocher C A and Commins E D 1967 Phys. Rev. Lett. 18575

[2] Clauser J F, Horne M A, Shimony A and Holt R A 1969 Phys. Rev. Lett. 23880

[3] Freedman S J and Clauser J F 1972 Phys. Rev. Lett. 28938

[4] Aspect A, Grangier P and Roger G 1981 Phys. Rev. Lett. 47460

Aspect A, Grangier P and Roger G 1982 Phys. Rev. Lett. 4991

[5] Huang H and Eberly J H 1993 J. Mod. Opt. 40915

[6] Muthukrishnan A, Agarwal G S and Scully M O 2004 Phys. Rev. Lett. 93093002

[7] Einstein A, Podolsky B and Rosen N 1935 Phys. Rev. 47777

[8] Ekert A K 1991 Phys. Rev. Lett. 67661

[9] Knill E, Laflamme R and Milburn G J 2001 Nature 40946

[10] Briegel H-J, Dur W, Cirac J I and Zoller P 1998 Phys. Rev. Lett. 815932

[11] Chaneliere T et al 2006 Phys. Rev. Lett. 96093604

[12] Benson O et al 2000 Phys. Rev. Lett. 842513

[13] Santori C et al 2001 Phys. Rev. Lett. 861502

[14] Akopian N et al 2006 Phys. Rev. Lett. 96130501

[15] Stevenson R M et al 2006 Nature 439179

[16] Young R J et al 2006 New J. Phys 829

[17] Hohenester U, Pfanner G and Seliger M 2007 Phys. Rev. Lett. 99047402

[18] Hudson A J et al 2007 Phys. Rev. Lett. 99266802

[19] Larque M, Robert-Philip I and Beveratos A 2008 Phys. Rev. A 77042118

[20] Gerardot B D et al 2007 Appl. Phys. Lett. 90041101

[21] Seguin A et al 2005 Phys. Rev. Lett. 95257402

[22] Avron J E et al 2008 Phys. Rev. Lett. 100120501

[23] Troiani F, Perea J I and Tejedor C 2006 Phys. Rev. B 74235310

[24] Johne R et al 2008 Phys. Rev. Lett. 100240404

[25] Gammon D et al 1996 Phys. Rev. Lett. 763005

[26] Kulakovskii V D et al 1999 Phys. Rev. Lett. 821780

[27] Agarwal G S 1974 Quantum Statistical Theories of Spontaneous Emission and their Relation to Other Approaches (Springer Tracts in Modern Physics) (Berlin: Springer) p 40

[28] Lax M 1968 Phys. Rev. 172350 